\title{
Modelo de suporte à decisão aplicado ao acesso a fisioterapia de indivíduos com Acidente Vascular Encefálico
}

\author{
Decision support model applied to access to physiotherapy in individuals affected by \\ cerebrovascular accident
}

Modelo de apoyo a la decisión aplicado al acceso a fisioterapia de individuos con accidente cerebrovascular

Luciana Moura Mendes de Lima ${ }^{1 *}$, Kátia Suely Queiroz Silva Ribeiro¹, Ronei Marcos de Moraes ${ }^{1}$.

\section{RESUMO}

Objetivo: Elaborar um modelo de suporte à decisão para indicar a necessidade do acesso aos serviços de fisioterapia aos indivíduos acometidos por Acidente Vascular Encefálico (AVE). Métodos: A pesquisa foi caracterizada por um recorte de um estudo longitudinal com indivíduos acometidos por AVE, residentes nas cidades de João Pessoa e Cabedelo, no estado da Paraíba, admitidos em um hospital público de referência da região. Resultados: Foi usado um modelo de suporte à decisão baseada em Redes Neurais Artificiais do tipo feedforward, e perceptron de múltiplas camadas. Ela foi treinada de modo supervisionado usando o algoritmo backpropagation. Constituída por 15 neurônios na camada de entrada, duas camadas ocultas com dois neurônios cada uma e uma camada de saída com dois neurônios. Foi verificado um percentual de acerto de 97,4\%. Conclusão: O modelo desenvolvido permitiu indicar a necessidade dos indivíduos acometidos por AVE ao acesso aos serviços de fisioterapia. Portanto, esse modelo pode auxiliar os profissionais de saúde nessa tomada de decisão.

Palavras-Chave: Acidente Vascular Cerebral, Tomada de Decisão, Redes Neurais (Computação), Acesso aos Cuidados de Saúde.

\begin{abstract}
A decision support model was build in order to indicate the needs for access to physiotherapy services for individuals affected by cerebrovascular accident (stroke). Methods: The research was characterized by a longitudinal study cutout of individuals affected by stroke living in the cities of João Pessoa and Cabedelo, in Paraíba State, who were admitted to a reference public hospital in the region. Results: A decision support model based on Artificial Neural Networks, feedforward type and multilayer perceptron. It was trained using a supervised backpropagation algorithm. It was composed by 15 neurons in the input layer, two hidden layers with two neurons each and an output layer with two neurons. Its accuracy was measured as $97.4 \%$. Conclusion: The decision model developed was able to indicate the access needs for individuals affected by stroke to physiotherapy services. So, this model can help health professionals in this decision making.
\end{abstract}

Keywords: Decision Making, Stroke, Neural Networks (Computer), Health Services Accessibility.

\section{RESUMEN}

Objetivo: Desarrollar un modelo de apoyo a la decisión para indicar la necesidad de acceso a servicios de fisioterapia para personas con accidente cerebrovascular. Métodos: La investigación es del tipo corte longitudinal. La población de estudio fue individuos con accidente cerebrovascular que vivían en las ciudades

${ }^{1}$ Universidade Federal da Paraíba (UFPB), João Pessoa-Paraíba. *E-mail: lucianamm_@hotmail.com 
de João Pessoa y Cabedelo, estado de Paraíba, ingresados en un hospital público de referencia en la región. Resultados: Se utilizó un modelo de apoyo a la decisión basado en redes neuronales artificiales de feedforward, perceptrón multicapa con entrenamiento supervisado de backpropagation. Consta de 15 neuronas en la capa de entrada, dos capas ocultas con dos neuronas cada una y una capa de salida con dos neuronas. Se verificó un porcentaje de aciertos del 97,4\%. Conclusión: El modelo desarrollado indicó la necesidad de que los pacientes con accidente cerebrovascular accedan a los servicios de fisioterapia. Por lo tanto, este modelo puede ayudar a los profesionales de la salud en esta toma de decisiones.

Palabras clave: Toma de Decisiones, Accidente Cerebrovascular, Redes Neurales (Computación), Accesibilidad a los Servicios de Salud.

\section{INTRODUÇÃO}

O Acidente Vascular Encefálico (AVE) é uma patologia causada pela interrupção no suprimento sanguíneo do encéfalo. Podendo ser classificado em isquêmico, quando há interrupção do fornecimento de sangue ao encéfalo e hemorrágico, no caso de ruptura de uma das artérias. Adicionalmente, podem variar conforme populações de diferentes idades, raças, etnias e nacionalidades (HANKEY GJ, 2017).

Esta doença representa um grande impacto na saúde pública mundial por ser a segunda principal causa mundial de óbitos (NAGHAVI M, et al., 2015) e a terceira de incapacidade prolongada em adultos (FEIGIN VL, et al., 2014).

As consequências do AVE podem estar relacionadas a déficit motor, sensorial, comprometimento cognitivo, linguagem, comunicação e limitações nas atividades de vida diárias (HEBERT D, et al., 2016). Cerca de $24 \%$ a $49 \%$ dos indivíduos que sobrevivem ao AVE apresentam algum grau de incapacidade (CARMO JF, et al., 2015). De acordo com Utida KAM, et al. (2016), grande parte dos pacientes com AVE admitidos em um centro de reabilitação apresentaram dependência total ou grave, enquanto no período da alta, estes em sua maioria apresentaram dependência leve ou independência total.

O indivíduo acometido por AVE necessita acessar os serviços de saúde que oferecem assistência a reabilitação, como exemplo, os serviços de fisioterapia. O acesso representa a ponte entre o cidadão e os serviços de saúde (SANTOS L e ANDRADE LOM, 2012).

A pesquisa de Sousa FOS, et al. (2014) afirma que a demora no agendamento das consultas e a falta de critérios de prioridades são aspectos que restringem o acesso aos serviços de saúde, dessa maneira demonstra um acesso fragmentado e pouco resolutivo. Em relação à fisioterapia não é diferente, uma investigação verificou que apenas $30,8 \%$ dos indivíduos acometidos por AVE tiveram acesso aos serviços de fisioterapia após três meses do acometimento (MENDES LM, et al., 2016).

Desarte, considerando a necessidade de garantir o acesso das pessoas acometidas por AVE aos serviços de fisioterapia é necessário propor estratégias e tomar decisão visando superar as dificuldades. Perante a problemática, o presente estudo teve como objetivo elaborar um modelo de suporte à decisão para indicar a necessidade do acesso aos serviços de fisioterapia aos indivíduos acometidos por AVE.

\section{MÉTODOS}

É um recorte do estudo longitudinal observacional, seguimento de indivíduos com AVE, e executado por meio de quatro fases de inquérito domiciliar. A população foi composta por indivíduos de ambos os sexos que deram entrada em um hospital público de referência da região, localizado em João Pessoa - Paraíba, como causa primária no Boletim de Emergência o AVE (isquêmico ou hemorrágico) entre os meses de abril e maio de 2013 e residiam nas cidades de João Pessoa e Cabedelo (MENDES LM, et al., 2016).

Para o estudo foram empregadas informações referentes as primeiras duas fases, a primeira entrevista, efetivada entre 15 e 21 dias após alta hospitalar (T0) e a segunda (T1), entre 90 e 105 dias após T0. Os dados 
referentes à identificação dos indivíduos foram fornecidos pelo Serviço de Arquivo Médico e Estatístico do hospital, em seguida uma equipe de pesquisadores entrava em contato com os sujeitos e/ou seus responsáveis legais, informando a finalidade da pesquisa e convidando-os a participar. As visitas domiciliares eram agendadas com antecedência via telefone. Foi verificado um total de 107 indivíduos em que 27 evoluíram ao óbito entre a alta e o momento de contato para o agendamento da primeira entrevista, cinco não aceitaram participar, 23 não foram identificados por endereço, telefone e/ou apresentaram dificuldade no agendamento da entrevista no prazo estabelecido do estudo, dois passaram a morar em outra cidade, findando em 50 indivíduos. Em virtude das perdas por alteração de endereço (5), nova recusa (1) e óbitos (5), após três meses da primeira entrevista, a população da pesquisa totalizou 39 indivíduos.

Os instrumentos aplicados na coleta dos dados foram desenvolvidos a partir de uma revisão da literatura. Para o estudo foi utilizado questões referentes à condição clínica, a presença de fatores risco para o AVE, comprometimento funcional por meio da Escala Modificada de Rankin (RANKIN J, 1957; VAN SWIETEN JC, et al., 1988) e o acesso aos serviços de fisioterapia. Foram selecionadas as seguintes variáveis: tipo de AVE, lado afetado, tempo transcorrido dos primeiros sinais (tempo de atendimento), número de episódios de AVE, Hipertensão Arterial Sistêmica (HAS), doença cardíaca, Diabetes Mellitus (DM), dislipidemia, etilismo, tabagismo, AVE prévio ou ataque isquêmico transitório, fibrilação atrial, Infarto Agudo do Miocárdio (IAM), fisioterapia no âmbito hospitalar e Escala Modificada de Rankin, tendo em vista serem as mais importantes com relação ao objeto de investigação.

Os dados foram armazenados em uma planilha eletrônica e analisados no software Waikato Environment for Knowledge Analysis (WEKA), versão 3.7.8. Este foi elencado em virtude de apresentar código aberto, possuir vários algoritmos de modelos de decisão. Alguns modelos estatísticos foram testados, tais como: Regressão Logística, Rede Naive Bayes e Árvore de Decisão. No entanto, as Redes Neurais Artificiais (RNA) foram escolhidas por apresentar os melhores resultados na decisão e quando comparado aos demais métodos citados. Diferentes configurações foram analisadas, mas a mencionada a seguir foi a melhor opção.

As RNA são sistemas de processamento paralelo, motivado na biologia humana, tendo o neurônio como principal elemento, sendo caracterizado como unidade de processamento da informação (Figura 1). Este método tem a capacidade de atuar em dados e situações complexas (HAYKIN S, 2001). Em geral, as RNA apresentam a seguinte configuração: uma camada de entrada, uma ou várias camadas ocultas e uma camada de saída. A camada de entrada tem a função de representar a distribuição dos dados de entrada para cada neurônio da camada seguinte; a oculta torna a rede capaz de extrair estatísticas e realizar o processamento necessário e a camada de saída indica a conclusão final (LUDWIG JR O e COSTA EMM, 2007).

Figura 1 - Representação de um neurônio artificial

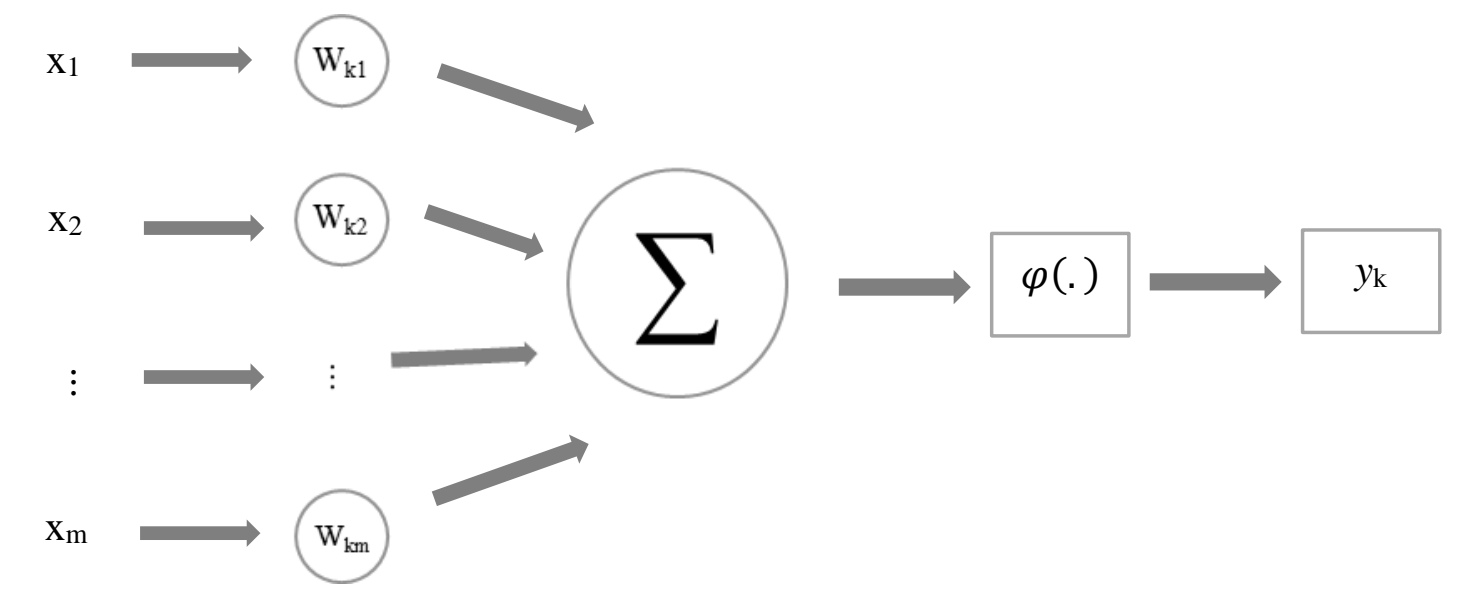

Fonte: Lima LMM, Ribeiro KSQS, Moraes RM, 2019. Adaptado Haykin S, 2001.

onde: $x_{m}$ é o sinal de entrada do neurônio; $W_{k m}$ é o peso sináptico do neurônio $k$; $\Sigma$ é o somador; $\varphi($.) é a função de ativação; $y_{k}$ é o sinal de saída do neurônio. 
Uma rede é caracterizada pela sua função de ativação, pelo padrão de suas conexões entre os neurônios (arquitetura da rede) e o seu método para determinar os pesos sobre as conexões (forma de aprendizagem). A função de ativação estabelece a forma e a intensidade de alteração dos valores transmitidos de um neurônio a outro. A arquitetura depende do problema a ser solucionado podendo ser classificadas pelo número de camadas e pelos tipos de conexões. Enquanto, a forma de aprendizagem é o processo de ajuste dos pesos sinápticos. Para o presente estudo foi elencado a função de ativação sigmoide, as redes multicamadas que apresentam neurônios que estão dispostos em várias camadas entre os nós de entrada e de saída e o tipo feedforward em que a partir da camada de entrada os sinais se propagam. A forma de aprendizagem foi supervisionada (HAYKIN S, 2001; LUDWIG JR O e COSTA EMM, 2007).

O modo de treinamento escolhido foi supervisionado do tipo backpropagation, algoritmo que atualiza os pesos da rede de maneira que a função de erro decresça progressivamente (HAYKIN S, 2001; LUDWIG JR O e COSTA EMM, 2007).

Dentre os tipos de RNA, o perceptron de múltiplas camadas ou multilayer perceptron (MLP) é uma rede composta por camada de entrada ou sensorial, uma ou mais camadas ocultas e uma camada de saída. Adicionalmente, treinada de forma supervisionada por meio da regra de aprendizagem que minimiza o erro e o sinal de entrada que se propaga para frente (HAYKIN S, 2001; LUDWIG JR O e COSTA EMM, 2007). Este erro pode ser calculado da seguinte maneira:

$$
e_{j}(m)=d_{j}(m)-y_{j}(m)
$$

onde: $e_{j}(m)$ é o sinal de erro de um neurônio de saída j na iteração $m ; d_{j}(m)$ é a resposta desejada para o neurônio j; $y_{j}(m)$ é o sinal de saída do neurônio j.

Para avaliar os resultados gerados pela RNA foi usado a matriz de confusão e o coeficiente Kappa. A matriz de confusão é uma matriz quadrada cujo a diagonal principal representa as decisões corretas e fora dela, as incorretas. Tem o intuito de verificar a precisão do modelo (FOODY GM, 2002). Contudo, o coeficiente Kappa tem o objetivo de mensurar o grau de concordância entre as taxas de acertos e erros alcançados, utilizando a matriz de confusão (COHEN J, 1960). Os valores do coeficiente Kappa podem ser classificados de acordo com o grau de concordância de Landis JR e Koch GG (1977).

O projeto foi submetido à avaliação pelo Comitê de Ética em Pesquisa do Centro de Ciências da Saúde, da Universidade Federal da Paraíba, tendo sido aprovado com o número do protocolo 0372/12 (CAEE: 06573712.9.0000.5188), respeitando os critérios estabelecidos na Resolução ํㅜ 466/12 de 12 de dezembro de 2012, que regulamenta a pesquisa em seres humanos.

\section{RESULTADOS E DISCUSSÃO}

A população do presente estudo, 39 indivíduos com AVE, apresentou uma distribuição homogênea em relação ao sexo, com faixa etária predominante a partir dos 60 anos $(53,8 \%)$. Em relação aos aspectos clínicos, a maioria teve AVE isquêmico $(69,2 \%)$, até dois episódios de AVE $(87,2 \%)$, sendo o lado direito mais afetado (46,2\%) em relação ao lado esquerdo e ambos (MENDES LM, et al., 2016).

Conforme a Escala Modificada de Rankin, a maioria dos indivíduos apresentou deficiência entre moderada e grave $(66,7 \%)$ no momento da primeira entrevista.

Dos entrevistados, $66,7 \%$ eram alfabetizados, $56,4 \%$ não tinha união estável, em sua maioria residiam com até quatro pessoas no domicílio $(74,4 \%)$, com uma renda familiar de até dois salários mínimos $(59,0 \%)$ e estavam inativos representando, $84,6 \%$ da amostra.

Em relação aos fatores de risco foi constatado que $82,1 \%$ dos indivíduos com AVE declaram ter o diagnóstico de HAS, 30,8\% DM e 43,6\% dislipidemia.

O consumo excessivo de álcool e o hábito de fumar foram relatados por $41,0 \%$ e 53,8\% da amostra, respectivamente. Além disso, 20,5\% auto referiram ter doença cardíaca; 20,5\% ter fibrilação atrial; $12,8 \%$ teve IAM ou cirurgia cardíaca e $43,6 \%$ AVE ou ataque isquêmico transitório (Tabela 1). 
Tabela 1- Fatores de risco dos indivíduos com Acidente Vascular Encefálico, João Pessoa e Cabedelo, Paraíba, 2013.

\begin{tabular}{|c|c|c|}
\hline Fatores de risco & $\mathbf{n}$ & $\%$ \\
\hline \multicolumn{3}{|c|}{ Hipertensão Arterial Sistêmica } \\
\hline $\operatorname{sim}$ & 32 & 82,1 \\
\hline Não & 7 & 17,9 \\
\hline \multicolumn{3}{|l|}{ Diabetes Mellitus } \\
\hline Sim & 12 & 30,8 \\
\hline Não & 27 & 69,2 \\
\hline \multicolumn{3}{|l|}{ Dislipidemia } \\
\hline Sim & 17 & 43,6 \\
\hline Não & 22 & 56,4 \\
\hline \multicolumn{3}{|l|}{ Etilismo } \\
\hline Sim & 16 & 41,0 \\
\hline Não & 23 & 59,0 \\
\hline \multicolumn{3}{|l|}{ Tabagismo } \\
\hline $\operatorname{Sim}$ & 21 & 53,8 \\
\hline Não & 18 & 46,2 \\
\hline \multicolumn{3}{|l|}{ Doença cardíaca } \\
\hline $\operatorname{sim}$ & 8 & 20,5 \\
\hline Não & 31 & 79,5 \\
\hline \multicolumn{3}{|l|}{ Fibrilação atrial } \\
\hline Sim & 8 & 20,5 \\
\hline Não & 31 & 79,5 \\
\hline \multicolumn{3}{|c|}{ Infarto Agudo do Miocárdio ou cirurgia cardíaca } \\
\hline $\operatorname{Sim}$ & 5 & 12,8 \\
\hline Não & 34 & 87,2 \\
\hline \multicolumn{3}{|c|}{ AVE ou Ataque Isquêmico Transitório } \\
\hline Sim & 17 & 43,6 \\
\hline Não & 22 & 56,4 \\
\hline Total & 39 & 100,0 \\
\hline
\end{tabular}

Fonte: Lima LMM, Ribeiro KSQS, Moraes RM, 2013.

Diversos fatores podem influenciar a ocorrência do AVE, sendo modificáveis ou não modificáveis. Os fatores modificáveis podem ser HAS, DM, tabagismo, etilismo, obesidade, dislipidemia, fibrilação atrial e os não modificáveis são a idade, hereditariedade, sexo e raça (CASTRO JAB, et al., 2009; CHOUDHURY MSJH, et al., 2015). Tais fatores podem potencializar a ocorrência de novos episódios ou piorar a situação clínica e funcional dos pacientes, principalmente na presença de doenças crônicas degenerativas como: HAS, o principal fator de risco modificável, e DM (MEDEIROS CSP, et al., 2017; TRIGUEIRO ACQ e GAGLIARDI RJ, 2019). O tabagismo e o etilismo são descritos na literatura como importantes fatores de risco modificáveis (LIMA CMG, et al., 2015). Ainda, o AVE ou Ataque Isquêmico Transitório prévio apresentam um risco alto de um novo episódio nesses pacientes (HAUSER S e JOSEPHSON S, 2015). A identificação dos fatores de risco é fundamental para o planejamento de ações de prevenção e promoção da saúde da população em geral. Embora muitos deles sejam conhecidos, a sua ocorrência em cada comunidade deve ser atenciosamente avaliada (COPSTEIN L, et al., 2013).

Em relação ao modelo de suporte à decisão foi baseado nas RNA feedforward, perceptron de múltiplas camadas com treinamento supervisionado do tipo backpropagation. Ela foi composta por 15 neurônios na camada de entrada, duas camadas ocultas com dois neurônios cada uma e uma camada de saída com dois neurônios. Foram testadas várias configurações, mas a referida anteriormente apresentou os melhores resultados na decisão e um elevado percentual de acerto. 
O modelo conseguiu indicar corretamente 38 indivíduos, correspondendo a 97,4\%, com apenas uma decisão errônea. A matriz de confusão, conforme pode ser observado no Quadro 1, apresentou os seguintes dados: dos 12 indivíduos com AVE que deveriam ter acesso aos serviços de fisioterapia, o modelo acertou em todos os casos, enquanto dos 27 indivíduos que não deveriam ter acesso, o modelo errou apenas em um indicando que ele deveria ter acesso. O coeficiente Kappa demonstrou 94,1\% de concordância configurando um modelo de decisão quase perfeito.

Quadro 1 - Matriz de confusão baseado nas Redes Neurais Artificiais.

\begin{tabular}{|l|c|c|}
\hline \multirow{2}{*}{$\begin{array}{l}\text { Verdadeira } \\
\text { decisão }\end{array}$} & \multicolumn{3}{|l}{$\begin{array}{l}\text { Decisão fornecida pelo modelo baseado em } \\
\text { RNA }\end{array}$} & Sim & Não \\
\cline { 2 - 3 } & 12 & 0 \\
\hline Sim & 1 & 26 \\
\hline Não & & \\
\hline
\end{tabular}

Fonte: Lima LMM, Ribeiro KSQS, Moraes RM, 2019.

Com a aplicação do modelo de suporte de decisão, RNA, foi possível verificar que todos os indivíduos com AVE que deveriam ter acesso aos serviços de fisioterapia tiveram, sendo de grande importância no processo de recuperação. Aqueles que não deveriam ter acesso o modelo erro em um caso afirmando que o indivíduo deveria ter acesso. Supõe-se que em virtude do seu perfil, a partir das variáveis estudadas, denotou esta necessidade. Do ponto de vista do paciente mesmo não tendo a necessidade do acesso não acarretará problemas para ele, entretanto para o serviço de fisioterapia está ocupando uma vaga de maneira dispensável. O modelo de suporte à decisão pode auxiliar os profissionais de saúde na indicação da necessidade ao acesso aos serviços de fisioterapia de indivíduos acometidos por AVE respaldado pelo alto grau de concordância do coeficiente Kappa.

O AVE é o maior contribuinte para os anos de vida ajustados por incapacidade neurológica global em 2016 (FEIGIN VL, et al., 2019). É uma população que necessita de uma maior atenção, pois milhões de pessoas após o AVE apresentam limitações nas atividades de vida diária e necessitam de reabilitação. A reabilitação deve acontecer de maneira precoce e integral. Mas o acesso a esses serviços é demorado, faltam critérios definidos para direcionar o usuário ao serviço de referência, não tem prioridade no acolhimento e existe uma desarticulação entre os serviços da atenção primária à saúde e da especialidade, impactando negativamente na qualidade do atendimento à população (SOUSA FOS, et al., 2014). No estudo desenvolvido por Miranda $\mathrm{RE}$, et al. (2018) os pacientes apontaram que as principais dificuldades para acessar o serviço de fisioterapia foram questões burocráticas e a falta de vagas. Desta forma, as RNA podem ser um importante aliado na tomada de decisão dos profissionais de saúde no âmbito dos indivíduos acometidos por AVE com ênfase no acesso aos serviços de fisioterapia.

Na literatura científica as RNA foram empregadas em indivíduos com AVE como objeto de estudo em diferentes objetivos. Uma investigação aplicou a RNA e outros métodos estatísticos (Regressão Logística e Análise Discriminante) para avaliar o desempenho em predizer a mortalidade nos primeiros dez dias após a lesão encefálica e verificou diferentes precisões em virtude do treinamento dos dados, dos métodos usados e dos tipos do AVE (ÇELIK G, et al., 2014).

Pesquisas que tiveram como intuito identificar com precisão as diferentes posturas funcionais (sentar, em pé, andar) de sujeitos com AVE (FULK GD, et al., 2012) e classificar a análise da marcha de indivíduos com AVE hemiparéticos e indivíduos saudáveis (SCHEFFER C e CLOETE T, 2012), utilizaram as RNA para tal, obtendo valores de acerto acima de $95 \%$ e $99,4 \%$, respectivamente. Em ambos os casos, com percentuais de acerto semelhantes ao presente estudo. As RNA também foram usadas para definir o programa adequado de reabilitação em pacientes com AVE (PRASERTSAKUL T, et al, 2014).

De acordo com Abedi $V$, et al. (2017), as RNA demonstraram ser uma ferramenta eficiente no reconhecimento da isquemia cerebral aguda e diferenciação do AVE isquêmico em um ambiente de emergência com um elevado percentual de precisão. Por conseguinte, as investigações que aplicaram as

REAS/EJCH | Vol.11(15) | e1391 | DOI: https://doi.org/10.25248/reas.e1391.2019 Página 6 de 9 
RNA em indivíduos acometidos por AVE foram voltadas para o diagnóstico, a mortalidade, a análise das posturas ou marcha, o planejamento do tratamento sem levar em consideração o acesso aos serviços de saúde, em especial a fisioterapia. Desse modo, reforça a potencialidade e utilidade da metodologia como também a relevância do estudo.

Adicionalmente, as RNA foram utilizadas para analisar as condições de acessibilidade das pessoas com deficiência em instituições de graduação em Enfermagem, no município de João Pessoa. As decisões propostas ao modelo foram com relação às condições de acessibilidade ser "bom" e "fraco" utilizando as RNA do tipo perceptron múltiplas camadas. Foi constatado que as condições de acessibilidade foram consideradas "fraco" e as RNA obtiveram $86 \%$ de acerto, demonstrando ser uma aliada no processo de tomada de decisões (PEREIRA FJR, et al., 2012). Sendo assim, apresenta similaridades da localidade com a presente investigação.

De acordo com Haykin S (2001) as RNA exibem propriedades e capacidades de uso importante, a saber: capacidade inapta de adaptar seus pesos sinápticos a mudanças do meio ambiente, potencial inerente de ser tolerante a falhas, capacidade de aprendizagem supervisionada, pode ser projetada para fornecer informação sobre a confiança ou crença na decisão tomada, entre outras. Outro aspecto examinado é que as RNA se apresentam como melhor alternativa em relação aos modelos tradicionais estatísticos (KACZMARCZYK K, et al., 2009; FONSECA RW, et al., 2012; ROZZA GL, et al., 2015). As redes de perceptron de múltiplas camadas com o algoritmo backpropagation foram empregadas de maneira eficaz em pesquisas com diferentes objetivos (SILVA CCS, et al., 2015; FERREIRA RP, et al., 2016) auxiliando na tomada de decisão.

Modelo de suporte a decisão utilizando RNA foi usado na área da saúde na previsão de riscos de cardiopata congênita em mulheres grávidas, identificação dos tipos de anomalias congênitas do sistema nervoso, previsão de depressão entre idosos e alocação de recursos financeiros da saúde (LI H, et al., 2017; LIMA LMM, et al., 2019; SAU A e BHAKTA I, 2017; ROSAS MA, et al., 2013). Foi possível perceber uma gama de evidências científicas na perspectiva de analisar problemas em saúde com sucesso.

A presente pesquisa apresentou algumas limitações: o modelo não permite conhecer as variáveis que apresentam maior ou menor influência no acesso. No que diz respeito ao tipo de estudo apresentou-se perdas por óbito, mudança de domicílio ou até mesmo falta de interesse dos sujeitos com AVE em participar ou continuar na pesquisa no decorrer do estudo e ainda o hospital público disponibilizou apenas dois meses para a realização da coleta dos dados, inviabilizando um número maior de indivíduos (MENDES LM, et al., 2016).

\section{CONSIDERAÇÕES FINAIS}

O estudo permitiu elaborar um modelo de suporte à decisão baseada em RNA, a partir das variáveis estudadas, e constatar a necessidade dos indivíduos acometidos por AVE ao acesso aos serviços de fisioterapia. Essas informações podem subsidiar os profissionais de saúde acerca da temática e auxiliar no processo de tomada de decisão. A RNA demonstrou a sua potencialidade no suporte à tomada de decisão e pode ser empregada para indicar o acesso em outros agravos. Como pesquisas futuras podem ser indicadas na ampliação do acesso a outros serviços de reabilitação.

\section{AGRADECIMENTOS E FINANCIAMENTO}

Apoio financeiro pela Coordenação de Aperfeiçoamento de Pessoal de Nível Superior (CAPES).

\section{REFERÊNCIAS}

1. ABEDI V, et al. Novel screening tool for stroke using artificial neural network. Stroke, 2017; 48(6): 1678-1681.

2. CARMO JF, et. Disability after stroke: a systematic review. Fisioterapia em Movimento, 2015; 28(2): 407-418.

3. CASTRO JAB, et al. Estudo dos principais fatores de risco para acidente vascular encefálico. Revista da Sociedade Brasileira de Clínica Médica, 2009; 7(3): 171-173. 
4. ÇELIK G, et al. Predicting 10-day Mortality in Patients with Strokes Using Neural Networks and Multivariate Statistical Methods. Journal of Stroke and Cerebrovascular Diseases, 2014; 23(6): 1506-1512.

5. CHOUDHURY MSJH, et al. Modifiable and non-modifiable risk factors of stroke: A review update. Journal of National Institute of Neurosciences Bangladesh, 2015; 1(1): 22-26.

6. COHEN J. A Coefficient of Agreement for Nominal Scales. Educational and psychological measurement, 1960; 20(1): 37-46.

7. COPSTEIN L, et al. Prevalence and risk factors for stroke in a population of Southern Brazil. Arquivos de neuropsiquiatria, 2015; 71(5): 294-300.

8. FEIGIN VL, et al. Global Burden of Diseases, Injuries, and Risk Factors Study 2010 (GBD 2010) and the GBD Stroke Experts Group. Global and regional burden of stroke during 1990-2010: findings from the Global Burden of Disease Study 2010. The Lancet, 2014; 383(9913): 245-255.

9. FEIGIN VL, et al. Global, regional, and national burden of neurological disorders, 1990-2016: a systematic analysis for the Global Burden of Disease Study 2016. The Lancet Neurology, 2019; 18(5): 459-480.

10. FERREIRA RP, et al. Study on daily demand forecasting orders using artificial neural network. IEEE Latin America Transactions, 2016; 14(3): 1519-1525.

11. FONSECA RW, et al. Modelos de predição da redução do consumo energético em edifícios que utilizam a iluminação natural através de regressão linear multivariada e redes neurais artificiais. Ambiente Construído, 2012; 12(1): 163175.

12. FOODY GM. Status of land cover classification accuracy assessment. Remote Sensing of Environment, 2002; 80(1): 185-201.

13. FULK GD, et al. Identifying Activity Levels and Steps in People with Stroke using a Novel Shoe-Based Sensor. Journal of Neurologic Physical Therapy, 2012; 36(2): 100-107.

14. HANKEY GJ. Stroke. The Lancet, 2017; 389(10069): 641-654.

15. HAUSER S, JOSEPHSON S. Neurologia Clínica de Harrison. 3. ed. Rio de Janeiro: McGraw-Hill, 2015.

16. HAYKIN S. Redes neurais: princípios e prática. 2nd ed. Porto Alegre: Bookman, 2001.

17. HEBERT D, et al. Canadian stroke best practice recommendations: stroke rehabilitation practice guidelines, update 2015. International Journal of Stroke, 2016; 11(4): 459-484.

18. KACZMARCZYK K, et al. Gait classification in post-Stroke patients using artificial neural networks. Gait \& posture, 2009; 30(2): 207-210.

19. LANDIS JR, KOCH GG. The measurement of observer agreement for categorical data. Biometrics, 1977; 33(1):159174.

20. LI H, et al. An artificial neural network prediction model of congenital heart disease based on risk factors: A hospitalbased case-control study. Medicine, 2017; 96(6): 1-7.

21. LIMA CMG, et al. Características epidemiológicas e clínicas dos pacientes acometidos por acidente vascular cerebral. Journal of the Health Sciences Institute, 2015; 33(1): 45-49.

22. LIMA LMM, et al. Identificação das anomalias congênitas baseado em um modelo de decisão a partir de redes neurais artificiais. Journal of Health Informatics, 2019; 11(1): 8-12.

23. LUDWIG JR O, COSTA EMM. Redes Neurais: Fundamentos e Aplicações com Programas em C. Rio de Janeiro: Editora Ciência Moderna Ltda, 2007.

24. MEDEIROS CSP, et al. Perfil social e funcional dos usuários da Estratégia Saúde da Família com Acidente Vascular Encefálico. Revista Brasileira de Ciências da Saúde, 2017; 21(3): 211-220.

25. MENDES LM, et al. Acesso de sujeitos pós-acidente vascular cerebral aos serviços de fisioterapia. Journal of Nursing UFPE, 2016; 10(2): 387-394.

26. MIRANDA RE, et al. Avaliação do acesso à fisioterapia após a alta hospitalar em indivíduos com Acidente Vascular Cerebral. Clinical \& Biomedical Research, 2018; 38(3): 245-252.

27. NAGHAVI M, et al. Global, regional, and national age-sex specific all-cause and cause-specific mortality for 240 causes of death, 1990-2013: a systematic analysis for the Global Burden of Disease Study 2013. The Lancet, 2015; 385(9963): 117-171.

28. PEREIRA FJR, et al. Condições de Acesso às Pessoas com Deficiência em Instituições de Ensino Enfermagem: Utilização de Redes Neurais Artificiais como suporte à decisão. Revista Brasileira de Ciências da Saúde, 2012; 16(2):143-148.

29. PRASERTSAKUL T, et al. Defining the rehabilitation treatment programs for stroke patients by applying Neural Network and Decision Trees models. In: The 7th 2014 Biomedical Engineering International Conference. IEEE, 2014: 1-5.

30. RANKIN J. Cerebral vascular accidents in patients over the age of 60: II. Prognosis. Scottish Medical Journal, 1957; 2(5): 200-215. 
31. ROSAS MA, et al. Uso das redes neurais artificiais na aplicação de metodologia para alocação de recursos da saúde. Revista de Saúde Pública, 2013; 47(1): 128-136.

32. ROZZA GL, et al. Estudo comparativo do uso de redes neurais artificiais e regressão linear múltipla para a previsão da concentração cáustica em uma etapa do processo de fabricação de alumina. Revista Produção Online, 2015; 15(3): 948-971.

33. SANTOS L, ANDRADE LOM. Acesso às ações e aos serviços de saúde: uma visão polissêmica. Ciência \& Saúde Coletiva, 2012; 17: 2876-2878.

34. SAU A, BHAKTA I. Artificial Neural Network (ANN) Model to Predict Depression among Geriatric Population at a Slum in Kolkata, India. Journal of Clinical and Diagnostic Research: JCDR, 2017; 11(5): 1-4.

35. SCHEFFER C, CLOETE T. Inertial motion capture in conjunction with an artificial neural network can differentiate the gait patterns of hemiparetic Stroke patients compared with able-bodied counterparts. Computer methods in biomechanics and biomedical engineering, 2012; 15(3): 285-294.

36. SILVA CCS, et al. Rede Neural Artificial e o Modelo de Apoio à decisão em Segurança Alimentar Nutricional. Journal of Nursing UFPE, 2015; 9(2):7078-7085.

37. SOUSA FOS, et al. Do normativo à realidade do Sistema Único de Saúde: revelando barreiras de acesso na rede de cuidados assistenciais. Ciência \& Saúde Coletiva, 2014; 19: 1283-1293.

38. TRIGUEIRO ACQ, GAGLIARDI RJ. Perfil clínico e funcional de pacientes acometidos por acidente vascular cerebral no município de Patos-PB. Temas em Saúde, 2019; 19(1):86-100.

39. UTIDA KAM, et al. Nível de independência funcional de pacientes após acidente vascular cerebral atendidos por equipe multiprofissional em uma unidade de reabilitação. Acta fisiátrica, 2016; 23(3): 107-112.

40. VAN SWIETEN JC, et al. Interobserver agreement for the assessment of handicap in Stroke patients. Stroke, 1988; 19(5): 604-607. 